\title{
Protein structure prediction using AI and quantum computers
}

Ben Geoffrey A S

ORCID : https://orcid.org/0000-0001-6545-6314

\begin{abstract}
This work seeks to combine the combined advantage of leveraging these emerging areas of Artificial Intelligence and quantum computing in applying it to solve the specific biological problem of protein structure prediction using Quantum Machine Learning algorithms. The CASP dataset from ProteinNet was downloaded which is a standardized data set for machine learning of protein structure. Its large and standardized dataset of PDB entries contains the coordinates of the backbone atoms, corresponding to the sequential chain of N, C_alpha, and $\mathrm{C}^{\prime}$ atoms. This dataset was used to train a quantum-classical hybrid Keras deep neural network model to predict the structure of the proteins. To visually qualify the quality of the predicted versus the actual protein structure, protein contact maps were generated with the experimental and predicted protein structure data and qualified. Therefore this model is recommended for the use of protein structure prediction using AI leveraging the power of quantum computers.
\end{abstract}

The code is provided in the following Github repository

https://github.com/bengeof/Protein-structure-prediction-using-AI-and-quantum-computers 


\section{Introduction}

The past few years have seen a surge in the application of data-driven AI methods to solve complex problems in biology [1-3]. The areas of application include genomics, proteomics, and drug discovery[4-10]. These have led to tremendous advances in research in biological science. Another emerging area from which life science research can leverage advances is through quantum computing[11-15]. Therefore this work seeks to combine the combined advantage of leveraging these emerging areas of Artificial Intelligence and quantum computing in applying it to solve the specific biological problem of protein structure prediction using Quantum Machine Learning algorithms. Quantum machine learning algorithms are machine/deep learning algorithms that leverage the power of quantum computers. Fault-tolerant quantum computers may be far off, however solving real-world quantum-chemistry problems on near-term quantum devices is possible through the Pennylane [16] which provides an interface to use any of the quantum hardware provided by any of the quantum hardware providers such as IBM, Google, or Microsoft. Classical data is encoded into quantum states through amplitude encoding techniques to reflect the quantum state/wave-function of the quantum circuit. The circuit parameters are then optimized to produce the solution. These quantum layers are introduced between classical Keras layers to turn classical Keras-based deep neural network into a quantum-classical hybrid deep neural network to solve data-driven AI problems through leveraging the power of quantum computers.

\section{Methods}

The CASP dataset from ProteinNet was downloaded. ProteinNet is a standardized data set for machine learning of protein structure [17]. Its large and standardized dataset of PDB entries contains the coordinates of the backbone atoms, corresponding to the sequential chain of $\mathrm{N}$, C_alpha, and C' atoms. This dataset was used to train a quantum-classical hybrid Keras deep neural network model whose model configuration is shown below in Fig. 1. A classical convolutional deep neural network inspired in part by Wang et. al. [18] along with introducing quantum Keras layers to make a hybrid model was built and the summary of input dimension, hidden layers, and output dimension of the network is shown in Fig.1.

The code is provided in the following Github repository https://github.com/bengeof/Protein-structure-prediction-using-AI-and-quantum-computers 
bioRxiv preprint doi: https://doi org/10.1101/2021.05.22 445242; this version posted May 24,2021 . The copyright holder for this preprint (which was not certified by peer review) is the author/funder, who has granted bioRxiv a license to display the preprint in perpetuity. It is made available under aCC-BY-NC-ND 4.0 International license.

Fig.1 - Model Configuration

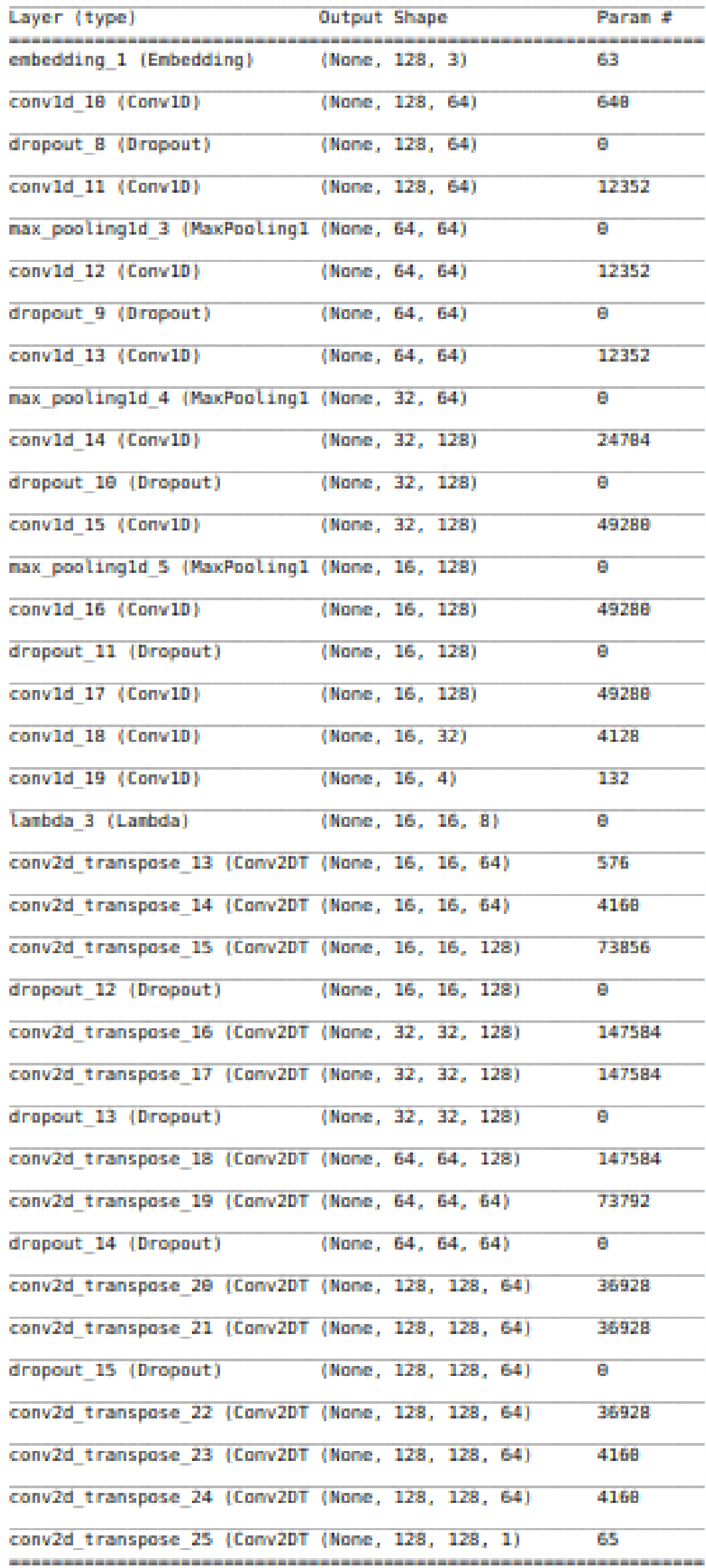


bioRxiv preprint doi: https://doi.org/10.1101/2021.05.22.445242; this version posted May 24, 2021. The copyright holder for this preprint (which was not certified by peer review) is the author/funder, who has granted bioRxiv a license to display the preprint in perpetuity. It is made available under aCC-BY-NC-ND 4.0 International license.

\section{Results and Discussion}

With the trained model, predictions were carried out. To visually qualify the quality of the predicted versus the actual protein structure, protein contact maps were generated with the actual and predicted protein structure data. The predicted and the actual contact map for two instances of prediction for a random seed is shown below in Fig. 2
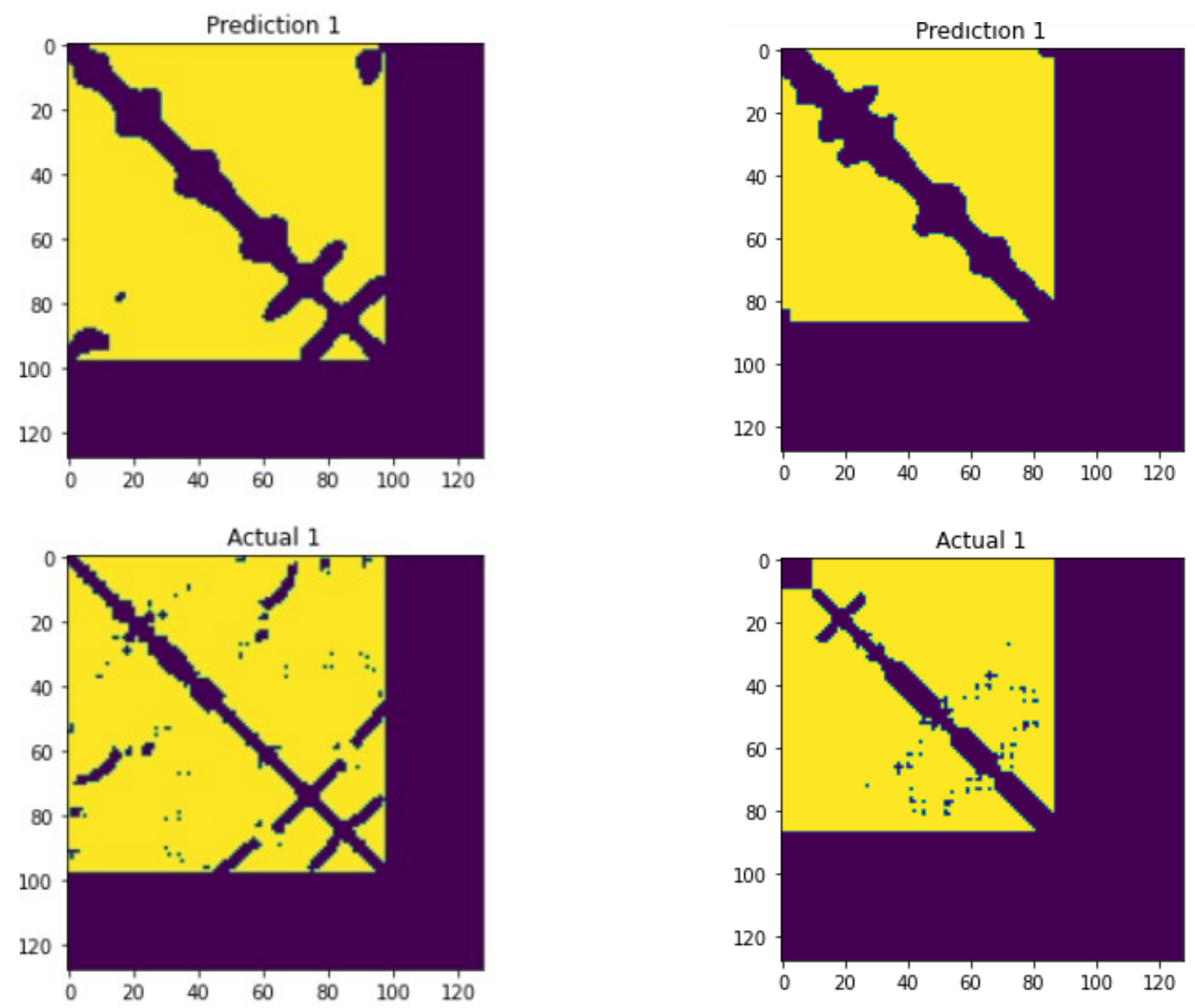

Fig. 2 - Protein contact maps

The converegnce of the model is depicted in the figure below

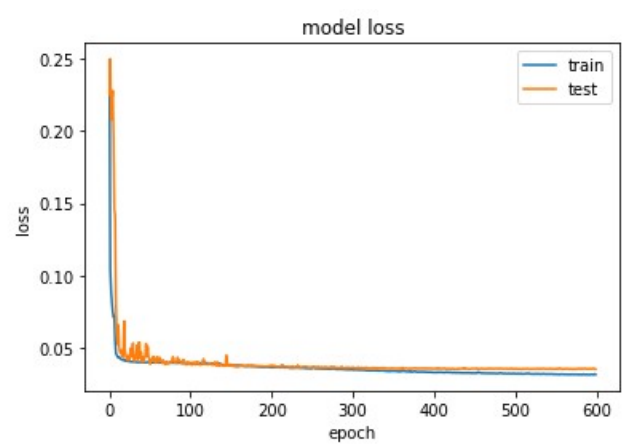

Fig. 3 - Loss function convergence 


\section{Conclusion}

This work seeks to combine the combined advantage of leveraging these emerging areas of Artificial Intelligence and quantum computing in applying it to solve the specific biological problem of protein structure prediction using Quantum Machine Learning algorithms. The CASP dataset from ProteinNet was downloaded which is a standardized data set for machine learning of protein structure. Its large and standardized dataset of PDB entries contains the coordinates of the backbone atoms, corresponding to the sequential chain of N, C_alpha, and $\mathrm{C}^{\prime}$ atoms. This dataset was used to train a quantum-classical hybrid Keras deep neural network model to predict the structure of the proteins. To visually qualify the quality of the predicted versus the actual protein structure, protein contact maps were generated with the experimental and predicted protein structure data and qualified. Therefore this model is recommended for the use of protein structure prediction using AI leveraging the power of quantum computers.

\section{Reference}

1. Tang, Binhua, Zixiang Pan, Kang Yin, and Asif Khateeb. "Recent advances of deep learning in bioinformatics and computational biology." Frontiers in genetics 10 (2019): 214

2. Mamoshina, Polina, Armando Vieira, Evgeny Putin, and Alex Zhavoronkov. "Applications of deep learning in biomedicine." Molecular pharmaceutics 13, no. 5 (2016): 1445-1454.

3. Akay, Altug, and Henry Hess. "Deep learning: current and emerging applications in medicine and technology." IEEE journal of biomedical and health informatics 23, no. 3 (2019): 906-920.

4. Zou, James, Mikael Huss, Abubakar Abid, Pejman Mohammadi, Ali Torkamani, and Amalio Telenti. "A primer on deep learning in genomics." Nature genetics 51, no. 1 (2019): 12-18.

5. Ramsundar, Bharath, Peter Eastman, Patrick Walters, and Vijay Pande. Deep learning for the life sciences: applying deep learning to genomics, microscopy, drug discovery, and more. " O'Reilly Media, Inc.", 2019.

6. Eraslan, Gökcen, Žiga Avsec, Julien Gagneur, and Fabian J. Theis. "Deep learning: new computational modelling techniques for genomics." Nature Reviews Genetics 20, no. 7 (2019): 389-403.

7. Chen, Hongming, Ola Engkvist, Yinhai Wang, Marcus Olivecrona, and Thomas Blaschke. "The rise of deep learning in drug discovery." Drug discovery today 23, no. 6 (2018): 12411250. 
8. Jing, Yankang, Yuemin Bian, Ziheng Hu, Lirong Wang, and Xiang-Qun Sean Xie. "Deep learning for drug design: an artificial intelligence paradigm for drug discovery in the big data era." The AAPS journal 20, no. 3 (2018): 1-10.

9. Dana, Dibyendu, Satishkumar V. Gadhiya, Luce G. St Surin, David Li, Farha Naaz, Quaisar Ali, Latha Paka et al. "Deep learning in drug discovery and medicine; scratching the surface." Molecules 23, no. 9 (2018): 2384

10. Urban, Gregor, Kevin Bache, Duc TT Phan, Agua Sobrino, Alexander K. Shmakov, Stephanie J. Hachey, Christopher CW Hughes, and Pierre Baldi. "Deep learning for drug discovery and cancer research: Automated analysis of vascularization images." IEEE/ACM transactions on computational biology and bioinformatics 16, no. 3 (2018): 1029-1035.

11. Cao, Yudong, Jhonathan Romero, and Alán Aspuru-Guzik. "Potential of quantum computing for drug discovery." IBM Journal of Research and Development 62, no. 6 (2018): 6-1.

12. Outeiral, Carlos, Martin Strahm, Jiye Shi, Garrett M. Morris, Simon C. Benjamin, and Charlotte M. Deane. "The prospects of quantum computing in computational molecular biology." Wiley Interdisciplinary Reviews: Computational Molecular Science 11, no. 1 (2021): e1481.

13. Barzen, Johanna, Frank Leymann, Michael Falkenthal, Daniel Vietz, Benjamin Weder, and Karoline Wild. "Relevance of Near-Term Quantum Computing in the Cloud: A Humanities Perspective." Cloud Computing and Services Science 1399 (2021): 25.

14. Alexeev, Yuri, Dave Bacon, Kenneth R. Brown, Robert Calderbank, Lincoln D. Carr, Frederic T. Chong, Brian DeMarco et al. "Quantum computer systems for scientific discovery." PRX Quantum 2, no. 1 (2021): 017001.

15. Li, Junde, Mahabubul Alam, Congzhou M. Sha, Jian Wang, Nikolay V. Dokholyan, and Swaroop Ghosh. "Drug Discovery Approaches using Quantum Machine Learning." arXiv preprint arXiv:2104.00746 (2021).

16. Bergholm, V., Izaac, J., Schuld, M., Gogolin, C., Alam, M.S., Ahmed, S., Arrazola, J.M., Blank, C., Delgado, A., Jahangiri, S. and McKiernan, K., 2018. Pennylane: Automatic differentiation of hybrid quantum-classical computations. arXiv preprint arXiv:1811.04968

17. AlQuraishi, Mohammed. "ProteinNet: a standardized data set for machine learning of protein structure." BMC bioinformatics 20, no. 1 (2019): 1-10

18. Wang, Sheng, Siqi Sun, Zhen Li, Renyu Zhang, and Jinbo Xu. "Accurate de novo prediction of protein contact map by ultra-deep learning model." PLoS computational biology 13, no. 1 (2017): $e 1005324$. 